\section{Organoids reveal cancer dynamics}

Single-cell analyses in cancer are limited by the small biomass of individual cells. In vitro production of 3D organoid structures from single tumour-derived cells generates sufficient biomass for in-depth analyses. SEE ARTICLE P.457

\section{CALVIN J. KUO \& CHRISTINA CURTIS}

$\mathrm{C}$ ancer is thought to arise from a single cell that proliferates to form a clonal population. Cells in that population then progressively acquire distinct mutations, leading to rampant tumour-cell diversification. Ideally, this intratumoral heterogeneity would be studied at the single-cell level - but such explorations are limited because single cells contain very little material for analysis. On page 457 , Roerink et al. ${ }^{1}$ overcome this limitation using methods for growing $3 \mathrm{D}$ cultures from single cells isolated from colorectal cancers. These organoid cultures can be used as proxies for the single cells from which they are derived, enabling in-depth analysis of tumour diversity.

Different studies of intratumoral heterogeneity have inferred distinct models of tumour evolution. According to the 'big bang' model, most detectable heterogeneity arises early in a cancer's evolution, coincident with the cells becoming cancerous. This is followed by proliferation of a range of subclones that are effectively equally fit ${ }^{2,3}$. In another model, tumours diversify gradually, through ongoing sequential selection of subclones that gain mutations conferring increased fitness ${ }^{3-5}$. Regardless of how it arises, heterogeneity is inextricably linked with intratumoral differences in growth, aggressiveness and sensitivity to therapy, so an in-depth understanding of the phenomenon would be valuable ${ }^{6,7}$.

Organoid techniques were initially developed to grow normal tissues in vitro in $3 \mathrm{D}$, but have proved equally useful for propagating clinical tumour specimens ${ }^{8-12}$. Roerink et al. set out to examine intratumoral heterogeneity using organoids. The authors took tissue from between four and six sites in tumours from three patients, and isolated single cells from each site (Fig. 1). Growth of these cells in specific culture conditions produced clonal organoids, in which all cells are nominally identical. In addition, the researchers grew clonal organoids from cells taken from the normal colorectal tissue surrounding each tumour, for comparison. The essentially unlimited proliferation of these single-cell-derived organoids generated sufficient material for deep genomic analysis, yielding numerous insightful conclusions.

The authors analysed the mutations in multiple cancer- and healthy-tissue-derived clonal organoids, and used this information to construct phylogenetic trees describing the lineage relationships between individual cells in a given tumour. In general, the trees recapitulated the geographic diversity of the sites from which the cells were taken. However, Roerink et al. found that clonal organoids from the same site were highly diverse, reflecting heterogeneity at the single-cell level.

The authors found mutations common genes APC, KRAS and TP53) in the trunks of the phylogenetic trees - that is, present in all cancer-derived organoids from a given person. But most mutations resided in the distal branches of the phylogenetic tree, arising after the cancer had undergone rapid proliferation. Overall, these results might be seen as providing support for the big-bang model in colorectal cancer, in which potential aggressiveness is determined by the early accrual of crucial driver mutations, rather than by the mutations that accumulate later in tumour growth ${ }^{2}$.

Next, Roerink et al. evaluated the mutational processes associated with tumour in colorectal cancer (such as those in the progression by analysing mutational signatures and then estimating the contribution of different signatures (which indicate different mutational processes) to the trunk and branches of phylogenetic trees. Certain mutational processes were acting in cancer cells but largely absent in healthy colon cells - and many more mutations were present in the cancer cells. the branches of an individual tumour, highlighting the contribution of different mutational processes across the tumour. On the basis of the mutational signatures and mutation burden, the authors inferred that the rates of both cell division and mutations per cell division were elevated in cancers compared with healthy cells. Consistent with an elevated mutation rate, the phylogenetic trees reveal rapid diversification in the tumour genome after the first cell becomes cancerous.

In addition to genomic data from each organoid, the authors profiled RNA transcripts and patterns of DNA modification by methyl groups (which can lead to altered gene expression). The way in which these profiles diversified across organoids closely traced the mutation-based phylogenies for each tumour. One key concern about organoid systems is that they cut cells off from the cues normally provided by the surrounding tissue - this might alter gene-expression or methylation patterns. That Roerink et al. observe stable diversification of these patterns indicates that tumour cells in organoids no longer rely on the surrounding microenvironment to maintain gene expression or methylation, highlighting the power of this experimental system.

The researchers' organoids also allowed them to analyse how mutational landscapes affect drug resistance. Certain targeted Mutational signatures also differed between

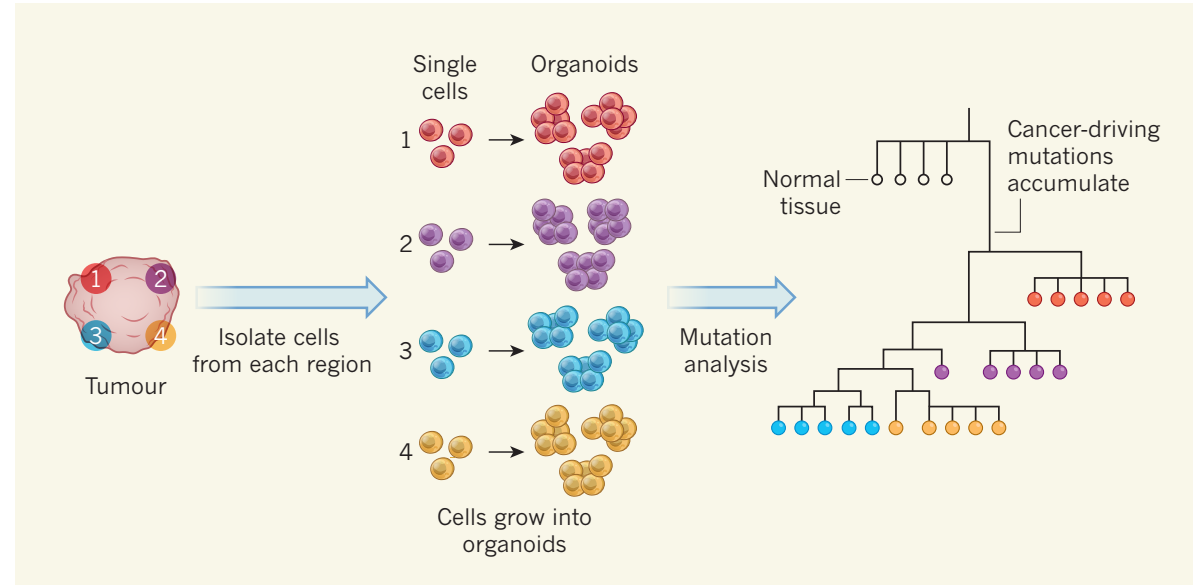

Figure 1 | Cancer analyses using organoids. Roerink et al. ${ }^{1}$ extracted tissue from several sites from human colorectal cancers. They induced single cells isolated from these samples, and from nearby healthy tissue (not shown), to grow in vitro to form 3D cultures called organoids. They then performed a range of analyses, including DNA sequencing, on each organoid. The authors used the mutations in each organoid to construct phylogenetic trees that showed lineage relationships between the organoids' genomes. Those derived from the same site (indicated by colour coding) bore most similarities. The analysis revealed that typical cancer-driving mutations arose in the trunk of the tree (that is, during early stages of tumour growth), but that diversification continued throughout tumour growth. (Tree adapted from Fig. 1 of ref. 1.) 
therapies acted as might be expected for instance, nutlin-3a, which inhibits an interaction between wild-type p53 protein (which is encoded by TP53) and a binding partner, effectively killed cells in organoids in which TP53 was not mutated. By contrast, sensitivity to general chemotherapies did not correlate well with mutational status. The heterogeneity of drug response among samples from different regions of the same tumour provides a particularly sobering demonstration of the challenges of therapeutic resistance in cancer.

Overall, Roerink and colleagues' study is a remarkable demonstration of the ability of organoid technologies to amplify rare cancer-cell populations and enable deep analyses. However, the current approaches have limitations. For instance, organoids do not provide a wholly accurate snapshot of the situation in a tumour, because the growth of organoids involves cell proliferation, which itself can lead to further mutations. To get around this problem, Roerink et al. focused on mutations present in all cells of an organoid in their analysis. Another caveat is that organoid culture methods might also select for cells particularly adapted for in vitro growth, potentially correlating with the most proliferative or aggressive cells.

Nevertheless, this and other organoid methods should in the future prove invaluable tools for the genetic and functional dissection of cancer. A big help in this endeavour will be biobanks of patient-derived organoids ${ }^{9,10,13}$, which have been established in the past few years to encapsulate the genetic diversity of cancers from different anatomical sites. Eventually, organoids might also incorporate components of the tumour microenvironment, enabling more-holistic cancer modelling. Finally, Roerink and colleagues' method could be generalized beyond cancer to enable the expansion of limiting amounts of biopsy tissue from inflammatory, metabolic or genetic disorders. Here, as in cancer, this approach could reveal cellular heterogeneity and inform treatment strategies.
Calvin J. Kuo is in the Division of Hematology, Stanford University School of Medicine, Stanford Cancer Institute, Stanford, California 94305, USA. Christina Curtis is in the Division of Oncology and the Department of Genetics, Stanford University School of Medicine, Stanford, California 94305, USA. e-mails: cjkuo@stanford.edu; cncurtis@stanford.edu

1. Roerink, S. F. et al. Nature 556, 457-462 (2018).

2. Sottoriva, A. et al. Nature Genet. 47, 209-216 (2015)

3. Sun, R. et al. Nature Genet. 49, 1015-1024 (2017).

4. Vogelstein, B. et al. Science 339, 1546-1558 (2013).

5. Nik-Zainal, S. et al. Cell 149, 994-1007 (2012).

6. Bivona, T. G. \& Doebele, R. C. Nature Med. 22 , 472-478 (2016).

7. Sharma, P., Hu-Lieskovan, S., Wargo, J. A. \& Ribas, A. Cell 168, 707-723 (2017).

8. Gao, D. et al. Cell 159, 176-187 (2014).

9. van de Wetering, M. et al. Cell 161, 933-945 (2015)

10.Boj, S. F. et al. Cell 160, 324-338 (2015).

11.Fujii, M. et al. Cell Stem Cell 18, 827-838 (2016).

12.Sachs, N. et al. Cell 172, 373-386 (2018).

13.Sato, T. et al. Gastroenterology 141, 1762-1772 (2011).

This article was published online on 11 April 2018.

\section{Transition states that allow cancer to spread}

\section{Cancers of epithelial-cell origin often contain some tumour cells that have acquired traits of mesenchymal cells. How this leads to cancer spread has now been illuminated in mouse models. SEE ARTICLE P.463}

\section{ERIK W. THOMPSON}

\section{\& SHIVASHANKAR H. NAGARAJ}

$\mathrm{I}$ $\mathrm{n}$ cancers that arise from epithelial cells, some of the tumour cells adopt features of mesenchymal cells and lose their epithelial characteristics. This phenomenon is known as the epithelial-to-mesenchymal transition (EMT), and the emergence of cells with mesenchymal features is often associated with a poorer prognosis for patients. How this transition occurs and the implications of EMT for metastasis, the process by which cancer spreads, are not fully understood. On page 463 , Pastushenko et al. ${ }^{1}$ report an analysis of mouse tumours undergoing EMT, revealing that distinct cell populations can be identified during this transition.

Cancers of epithelial tissues are called carcinomas and can give rise to cells with mesenchymal properties, such as an elongated shape. These mesenchymal cells have been implicated $^{2}$ in tumour metastasis. The EMT process is also associated with cancer stem cells found in breast cancer and other cancer types, and is linked to the presence of the circulating tumour cells in the bloodstream that are a hallmark of tumour-relapse risk ${ }^{3}$. In numerous mouse models ${ }^{2}$, prevention of EMT or its reversal - a process known as MET, in which mesenchymal cells transition back to epithelial cells - can reduce the stem-cell-like properties of tumour cells and thereby reduce their ability to metastasize.

Although some studies ${ }^{4,5}$ indicate that not all tumour metastases require EMT, considerable amounts of data implicate ${ }^{6}$ the process of MET as a requirement for metastatic cells to successfully colonize locations beyond the initial tumour site. One way to address this controversy would be to gain a better understanding of how the EMT process occurs.

A hybrid EMT state, also known as a metastable state or partial EMT, in which individual cells express both epithelial and mesenchymal markers, has often been observed in carcinoma ${ }^{7}$, developmental processes and wound healing ${ }^{8}$. One model for how EMT might occur is that cells make a gradual transition from one state to the other along a continuous spectrum of change in which cells lose epithelial characteristics and concurrently gain mesenchymal ones. This is consistent with gene-expression analysis of cell lines from various types of carcinoma ${ }^{9}$. However, computational-modelling studies ${ }^{7}$ and in vitro observations ${ }^{8}$ indicate instead that distinctive, stable, long-lasting cell populations could represent discrete intermediate stages of EMT.

Pastushenko and colleagues investigated whether stable EMT states in tumours could be identified in vivo. The authors used genetically engineered mice that provide a model system in which to study skin carcinoma and have tumour cells ${ }^{10}$ that are known to exhibit EMT. Pastushenko et al. studied cells that had lost expression of the epithelial-cell marker EpCAM, and analysed the expression of more than 170 cell-surface proteins to try to identify useful markers of cell subpopulations. They then focused on three of these markers (CD61, CD51 and CD106) that are characteristic of a mesenchymal-cell state. This enabled the authors to identify distinct cell populations that had lost expression of the epithelial-cell marker EpCAM and expressed different combinations of these three mesenchymal markers.

Using the three markers as a tool to enable the isolation of cell populations present when EMT occurs, the authors isolated and characterized six distinctive cell subpopulations that lacked EpCAM expression. The authors used an impressive array of biomolecular analyses to assess the cellular characteristics of these populations, including their metastatic traits and their state of cellular differentiation. This work provides in vivo evidence of stable cell populations representing intermediate EMT states.

The authors also identified comparable cell subpopulations, characterized by the same markers, in both a mouse mammarytumour model and human tumour samples transplanted into mice, suggesting that these subpopulations represent stable transitional cellular states found in different cancer types. 